\title{
Hemosiderotic Fibrohistiocytic Lipomatous Lesion: Ten Cases of a Previously Undescribed Fatty Lesion of the Foot/Ankle
}

\author{
Cristina Marshall-Taylor, M.D., Julie C. Fanburg-Smith, M.D. \\ Soft Tissue Pathology Department, Armed Forces Institute of Pathology, Washington DC (JCF-S); \\ Department of Pathology, Tufts University/New England Medical Center, Boston, Massachusetts (CM-T)
}

We address the clinicopathologic features of a previously undescribed heavily-pigmented spindle cell proliferation within a circumscribed benign lipomatous lesion that occurs mainly in the ankle region of older females. Patients with "lipoma with fibrohistiocytic proliferation" were retrieved from our files. Slides and clinical information were reviewed, and immunohistochemistry was performed $(n=5)$. Ten patients with hemosiderotic fibrohistiocytic lipomatous lesions were identified. All cases demonstrated a well-circumscribed fatty lesion with random focal proliferations of plump, slightly pleomorphic spindled cells, scattered inflammatory cells, and abundant iron pigment. The spindled cells had vesicular nuclei with indistinct nucleoli; occasional hyperchromatism was observed. No nuclear cytoplasmic inclusions were identified. The spindled component had a reactive appearance. In most cases, the fatty component, with homogeneously sized adipocytes, predominated. The lesions occurred in the foot/ankle region $(8 / 10$, one each cheek and hand) of primarily females $(8 / 10)$ with a mean age of 50.6 years (range 42-63 years), size of $7.7 \mathrm{~cm}$ (range $2.5-17 \mathrm{~cm}$ ), and prior duration of 3.1 years. Seven of eight patients had a history of prior trauma. The spindled component was positive for vimentin, calponin, CD34, and occasionally KP-1 or lysozyme and negative for caldesmon, S100, and desmin. Follow-up on eight patients revealed four with recurrences or residual disease over three years, requiring re-excision. No cases metastasized or caused patient death (mean 12 years, range 1-23

Copyright () 2000 by The United States and Canadian Academy of Pathology, Inc.

VOL. 13, NO. 11, P. 1192, 2000 Printed in the U.S.A.

Date of acceptance: June 8, 2000.

The opinions and assertions contained herein are those of the authors and are not to be construed as official or representing those of the Department of the Army or Department of Defense.

Address reprint requests to: Julie C. Fanburg-Smith, M.D., Armed Forces Institute of Pathology, Department of Soft Tissue Pathology, Room 3075, 14th Street and Alaska Avenue, NW, Washington, D.C. 20306-6000; e-mail: Fanburg@afip.osd.mil; fax: 202-782-9182. years). We describe a predominantly fatty lesion that is hemosiderin rich with a "fibrohistiocytic" proliferation, composed of histiocytes, myofibroblasts, and C34-positive fibroblasts, which occurs predominantly in the ankle region of middle-aged females. We believe that this is a reactive process due to antecedent trauma, the inflammatory cells, hemosiderin, mixed spindled cells, and homogeneous non-neoplastic appearance of the fat. HFLL can be distinguished from previously described lesions. Correct identification of hemosiderotic fibrohistiocytic lipomatous lesion is important, as it may locally recur.

KEY WORDS: Fibrohistiocytic, Histiocytic, Lipoma, Lipomatous neoplasm, Myofibroblastic, Pigmented, Soft tissue.

Mod Pathol 2000;13(11):1192-1199

A search for these lesions was initiated because one of the authors (JCF-S) had encountered in practice several circumscribed fatty lesions with a spindled proliferation that resembled fibrohistiocytic neoplasms. The overall features were unlike spindle cell lipoma or other lipomatous neoplasms and had too much fat as part of the lesion for other spindle cell tumors including fibrohistiocytic and myofibroblastic neoplasms. To our knowledge, there have been no previous reports of such an entity. These lesions were superficial, found mainly in the ankle region, and were primarily composed of fat. We considered the possibility that this lesion was neoplastic. However, it is unencapsulated and has homogenously sized adipocytes, similar to normal fat. Hemosiderotic fibrohistiocytic lipomatous lesion (HFLL) appears reactive, with its mixed spindled cell population, inflammatory infiltration, and heavy hemosiderin deposition. A history of prior trauma was elicited from many of the patients. Furthermore, superficial lipomas occur mainly on the trunk and are seldom encountered on the distal lower extremities and feet $(1,2)$. We wanted to 
explore the biological behavior and clinicopathologic features of this novel lipomatous entity.

\section{MATERIALS AND METHODS}

A search for lesions similar to those that one of the authors (JCF-S) had encountered in practice was performed. The cases had been coded as "lipoma with fibrohistiocytic proliferation." They were retrieved from the soft tissue registry of the Armed Forces Institute of Pathology (AFIP) and reviewed. Out of 24 potential cases, only 10 had the common features that are herein described. Two lesions, better classified as dermatofibrosarcoma protuberans (DFSP) or atypical lipoma, respectively, were excluded. Twelve cases of a distinctive true lipoma variant that lacked the features of the current entity were excluded. All patient histories of the included group were reviewed. Additional history was obtained from the patients or patients' physicians. Gender, age, race, size, site, duration of symptoms, medical history, history of regional trauma, family history, history of recurrences or lesion progression, and patient status were recorded and compared. Perl's iron, Fontana stain, and immunohistochemistry (Table 1) were performed on all cases with available unstained slides or blocks $(n=5)$. Immunohistochemical techniques were by $A B C$ method, using the 3,3'-diaminobenzidine chromogen.

\section{RESULTS}

\section{Clinical Findings}

Ten patients with this unusual entity were identified. The ratio of these patients with HFLL to all patients with benign lipomatous lesions (predominantly lipomas) in the AFIP files from 1966 to the present is less than $0.2 \%(10 / 5666)$. There were eight females and two males. The mean age was 50.6 years with an age range of 42 to 63 years. The most common location was the foot/ankle (eight patients, seven female and one male), followed by one each of the hand and cheek. Sites on the foot were specifically described as dorsal in location (two cases), posterior (one case), the "foot/ankle" or simply "ankle" (two cases), or the "posterior distal calf" (one case). In six cases, the exact location on the foot was unspecified or unobtainable; however, no case was reported to be plantar in location. The majority of patients were Caucasian $(n=7)$; there was one each of African American, Hispanic, and unknown ethnicity. Four patients had a family or personal history of high cholesterol or myocardial infarction. One patient had an additional classic lipoma. A history of trauma to the area of the lesion was obtained in seven of eight patients (88\%), within 3 years before development of their mass. Trauma included a specific injury and/or multiple repeated injuries. One patient had a $2 \times 4$ wood plank fall on her foot and always wore tight shoes. Another patient had a prior stress fracture of the foot and her shoes were uncomfortable because of her high arches. A third patient had banged her foot. A fourth patient stood on her feet a lot and did significant physical activity as a cleaning lady. A fifth patient, with a cheek lesion, had a long history of some type of prior inflammatory process of the cheek, thought to be a cyst. The sixth patient had smashed his hand on a pipe during a fire-fighting drill before development of the lesion. The seventh patient had a history of unspecified trauma to the area, mentioned in his patient chart. The one patient with no specific history of trauma to the left posterior distal calf had left-sided plantar fasciitis from daily long-distance walking before development of her ankle lesion, perhaps indicating possible repeated mechanical injury to the ankle area, as well. None of the patients reported vascular insufficiency or superficial ulcerations. Lesions were originally considered (by the contributing pathologist) to be fibromatosis, pseudosarcomatous fasciitis, fibrous histiocytoma, dermatofibrosarcoma protuberans, traumatized lipoma, fat necrosis, spindle cell lipoma, well-differentiated liposarcoma, or myxoid liposarcoma. Follow-up on eight

Table 1. Antibodies Used, Their Pretreatments, Dilutions, and Sources

\begin{tabular}{|c|c|c|c|}
\hline Antibody & Clone & Dilution, Pretreatment & Source \\
\hline Vimentin & V9 & $1: 1600$, microwave & Biogenex, San Ramon, CA \\
\hline CD34 & QBEND & $1: 80$, microwave & Biogenex \\
\hline Calponin & CALP & $1: 100$, pepsin digestion & DAKO, Carpinteria, CA \\
\hline S100 protein & Polyclonal & $1: 1600$, no pre-treatment & DAKO \\
\hline Melanoma antigen & HMB-45 & $1: 50$, no pre-treatment & DAKO \\
\hline EMA & E29 & $1: 40$, microwave & DAKO \\
\hline Cytokeratin & AE1/AE3 & $1: 2$, protease digestion & Signet, Dedham, MA \\
\hline SMA & $1 \mathrm{E} 7$ & $1: 8000$, no pre-treatment & Sigma, St. Louis, MO \\
\hline MSA & HHF-35 & $1: 1600$, no pre-treatment & Enzo, Farmingdale, NY \\
\hline Desmin & D33 & $1: 40$, microwave & DAKO \\
\hline H-caldesmon & h-CD & $1: 200$, microwave & DAKO \\
\hline Lysozyme & Polyclonal & 1:1000, protease digestion & DAKO \\
\hline CD68 & $\mathrm{KP}-1$ & $1: 400$, protease digestion & DAKO \\
\hline
\end{tabular}


of 10 patients over a mean of 12 years (range 1 to 23 years), revealed that four patients had local recurrence or residual disease within 3 years, requiring re-excision. No lesions metastasized or caused patient death. Clinical information and follow-up on the patients with hemosiderotic fibrohistiocytic lipomatous lesions are summarized in Table 2.

\section{Pathology}

Grossly, the lesions were described as "darker yellow than usual," "yellow-brown," or having "pigmentary change." A few lesions were described as fatty tissue with areas of white nodularity or mucoid change. The lesions measured $7.7 \mathrm{~cm}$ on average with a range of $2.5 \mathrm{~cm}$ to $17.0 \mathrm{~cm}$. All lesions were solitary. Microscopically, the lesions were subcutaneous and consisted of well-delineated fatty tissue (Fig. 1) with several quilt-like, focal proliferations of spindled, plump, slightly pleomorphic spindled cells, scattered inflammatory cells, and abundant iron pigment (Fig. 2). The nuclei of the spindled cells were vesicular with indistinct nucleoli. No case exhibited intranuclear vacuolar inclusions. Occasional hyperchromatism was observed. The spindle cells, inflammatory cell, and accompanying fibrosis proliferated along the septa, between the lobules of mature adipose tissue, and around blood vessels and individual fat cells (Fig. 3). Occasional larger nuclei were identified, but without significant atypia. Some cells resembled the floret cells of lipomatous tumors (Fig. 4). A mixed infiltrate of lymphocytes, plasma cells, histiocytes (even multinucleated ones), and mast cells (Fig. 4) characterized the inflammatory background. No ectatic or aneurysmal blood vessels were noted; however, two cases exhibited perivascular hyalinization around a few small to medium sized vessels, adjacent to the spindled proliferations. Granular brown-to-golden pigment was identified in all cases. Fontana and iron stains available on five cases demonstrated the pigment to be iron (Fig. 5). Some of the cases represented the recurrent tumor, but we did not have the original tumor for comparison. The cases that were recurrent were no different from the other primary cases; i.e., the recurrent cases were not more cellular than the primary cases. Immunohistochemical stains on paraffinembedded tissue showed the lesions to be focally positive for lysozyme (1/2) and KP1 (3/3) (Fig. 6). The spindled cells were positive for vimentin $(2 / 2)$, CD34 (3/3) and calponin (2/2) (Fig. 7) and negative for S100 (0/5), HMB45 (0/3), desmin (0/5), epithelial membrane antigen $(0 / 2)$, cytokeratins $(0 / 2)$, smooth muscle actin (0/5), muscle specific actin $(0 / 5)$, and caldesmon $(0 / 3)$. This staining pattern supported a mixed population of histiocytes, CD34positive fibroblasts, and myofibroblasts, all within fat, similar in morphology to fibrohistiocytic tumors or the spindled component of spindled lipomatous lesions. Control tumors revealed all spindled areas to be positive for CD34 but only one of 15 dermatofibrosarcoma protuberans (focally in the fibrosarcomatous transformation component), one of seven spindle cell lipomas, and zero of seven well-differentiated liposarcomas to be focally positive for calponin.

\section{DISCUSSION}

Hitherto designated "hemosiderotic fibrohistiocytic lipomatous lesion" (HFLL), this rare benign fibrolipomatous entity represents less than $0.2 \%$ of all benign lipomatous lesions at the AFIP over the last 24 years. It is a well-delineated lipomatous lesion with several scattered septal, periadipocytic, or perivascular proliferations of plump, slightly pleomorphic spindled cells associated with abundant iron pigmentation and scattered inflammation. Clinically, HFLL is typically found superficially on the ankle or foot of predominantly middle-aged (mean age, 50.6 years) women $(80 \%)$ with a history of antecedent trauma (88\%). Two of the women reported tight fitting, uncomfortable shoes, and this may represent a gender specific problem that could explain why this entity is more common in females than males. Although several patients had a history or family history of hypercholesterolemia or heart disease, this finding may be coincidental. Several of the lesions were very large (mean, $7.7 \mathrm{~cm}$; range, 1 to $17 \mathrm{~cm}$ ), larger than classic lipoma (mean, $3 \mathrm{~cm}$ ) $(1,2)$. It is possible that these lesions reached their large sizes because these patients did not seek medical attention for up to 7 years (mean, 3.1 years). Fifty percent of patients with follow-up experienced recurrent or residual local disease, which may be related to the large size of these lesions or possibly to repeated trauma.

We believe that this lesion should be classified as a lipomatous lesion because of the predominance of fat and the consistent circumscription. The adipocytes, however, are not heterogeneous, as seen in most fatty neoplasms, but are homogenous in size, resembling normal mature fat. The spindled cells in HFLL are a mixture of myofibroblasts, fibroblasts, and histiocytes with hemosiderin pigmentation that we choose to call collectively "fibrohistiocytic," especially because these spindled areas morphologically resemble fibrohistiocytic tumors.

The immunophenotype of the spindled area is mixed. The lesion coexpresses CD34, Calponin, and occasionally KP-1 or lysozyme. CD34 positivity is typically found in a subset of perivascular, intraneural, and periadnexal fibroblasts (2-5). Although other spindled lipomatous lesions and various fibroblastic 


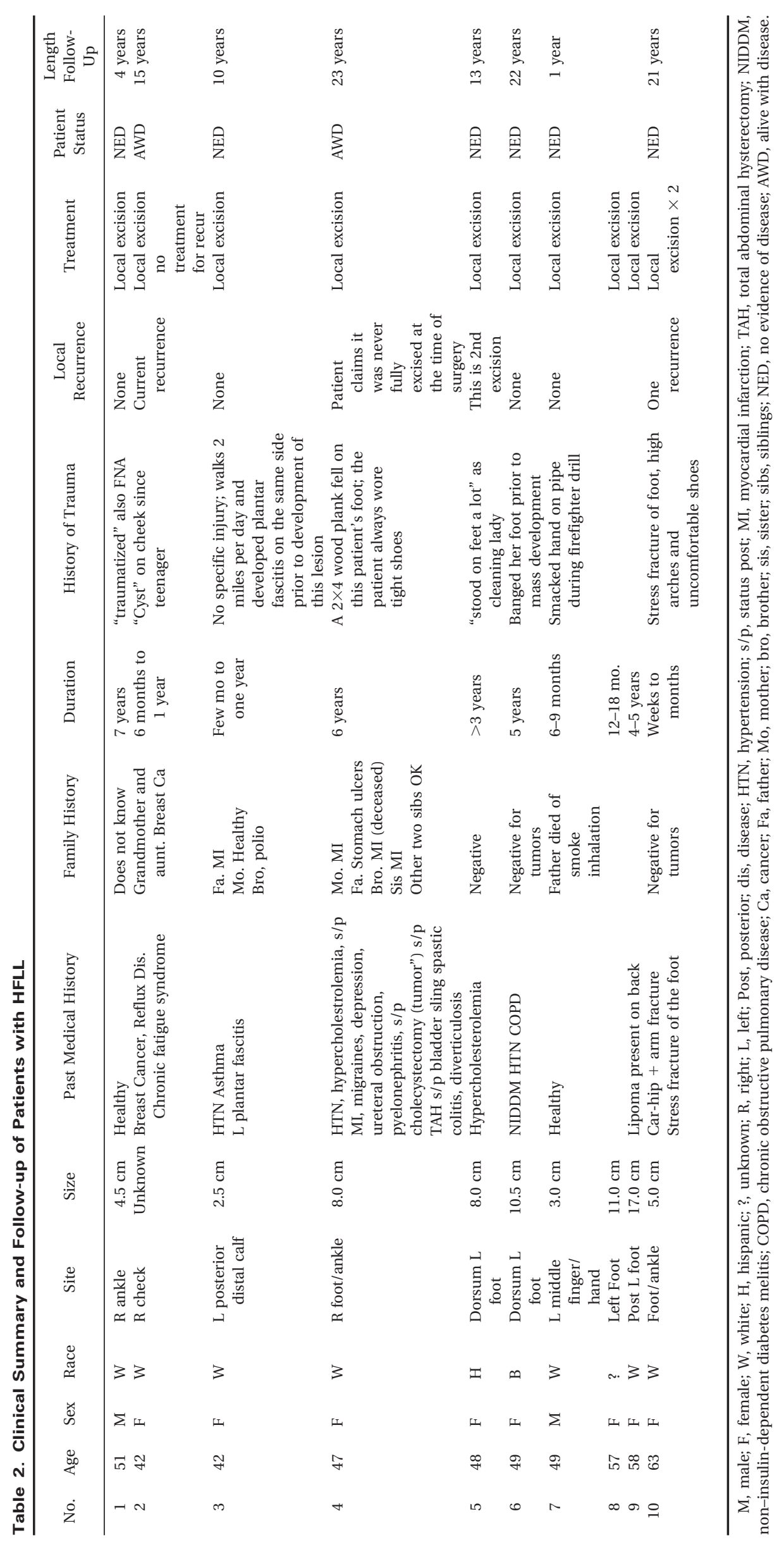




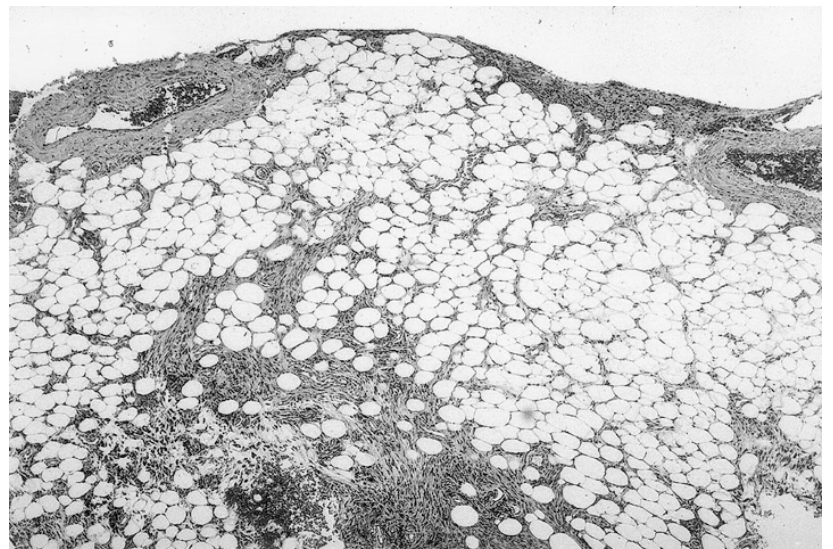

FIGURE 1. Hemosiderotic fibrohistiocytic lipomatous lesion (HFLL) with a circumscribed fatty component and a spindle cell component.

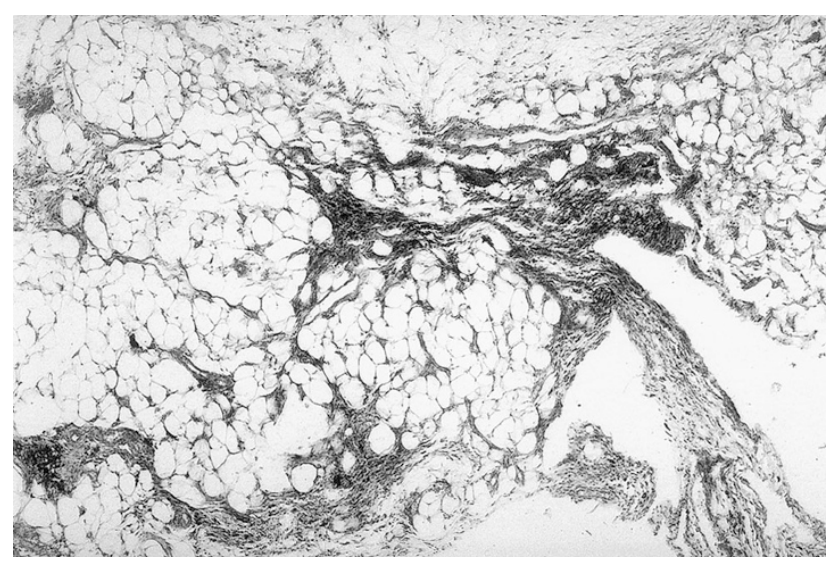

FIGURE 2. Distinctive pigment admixed with histiocytic, fibroblastic and myofibroblastic spindled cells, all within a circumscribed fatty component, is noted at lower magnification in HFLL.

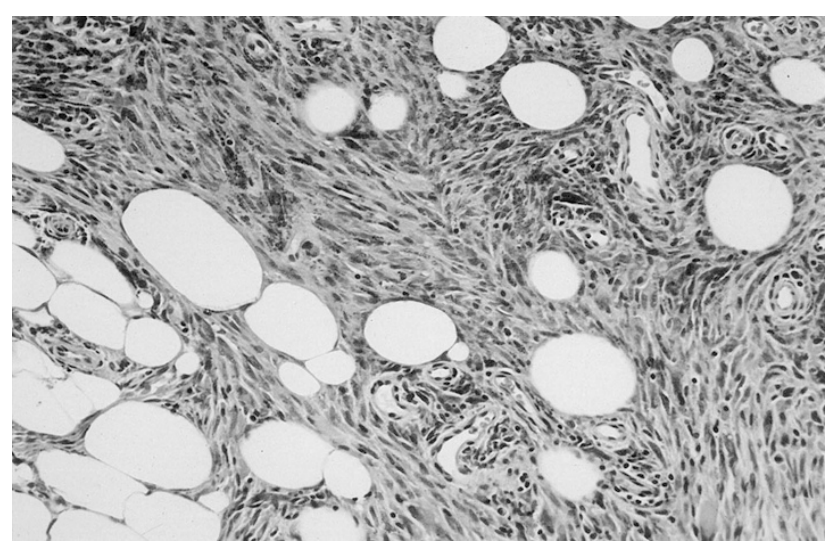

FIGURE 3. The spindled cells in HFLL are plump and slightly pleomorphic and run along fibrous septa and around individual adipocytes.

tumors are also positive for CD34 (2-5), the morphology, heavy pigment, and cellular composition differs from other spindled lipomatous lesions and the presence of abundant fat differs from other fibroblastic lesions. Coexpression of calponin (6-9) has not been extensively studied in CD34-positive spindled lipo-

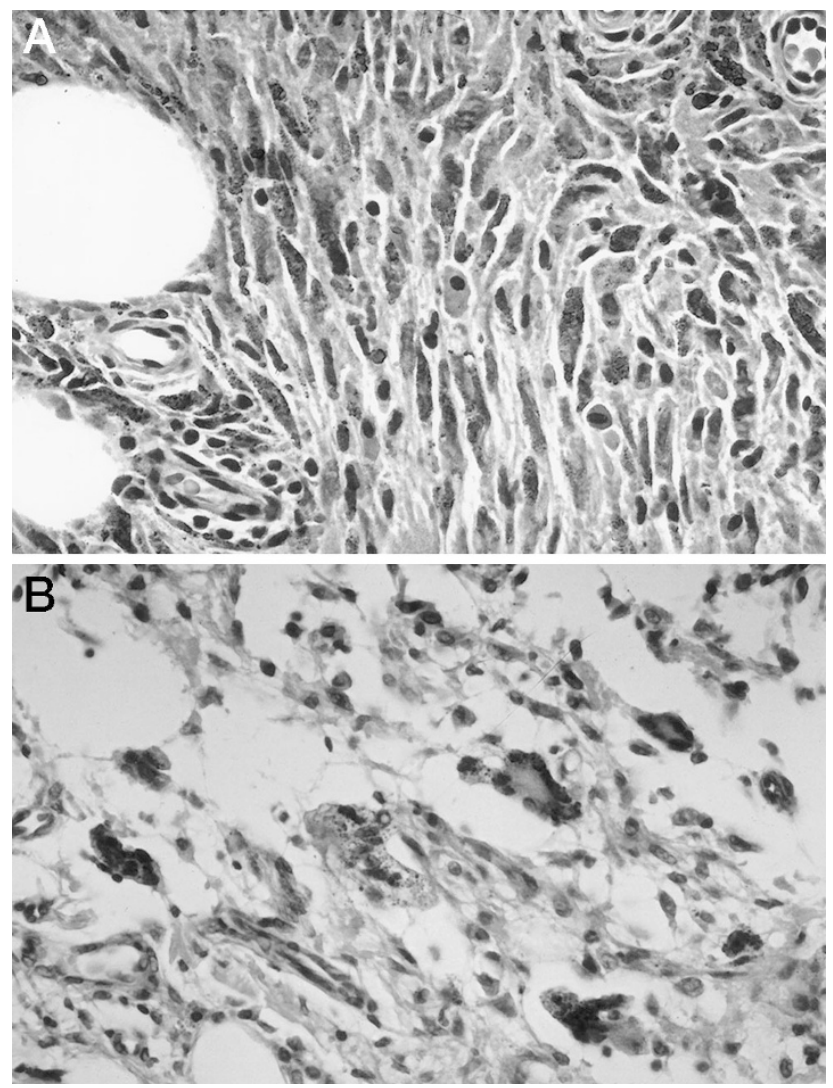

FIGURE 4. The inflammatory infiltrate among the spindled cells in HFLL includes mast cells (A) as well as pigmented histiocytes; some of the lesional cells resemble floret cells seen in lipomatous tumors (B).

matous tumors. Calponin is expressed in cells with contractile proteins, i.e., myofibroblasts, myoepithelial cells, and smooth muscle cells (6-9). It is positive in myofibroblastic tumors, fibrohistiocytic tumors (with myofibroblasts), myoepithelial tumors, and some gastrointestinal stromal tumors (10). Although calponin should theoretically stain similarly to muscle-specific actin, this is not the case in our experience. In fact, calponin appears to be more sensitive than actin for myofibroblasts or myoid cells. For example, angiomatoid "malignant" fibrous histiocytoma was found to be positive for calponin and desmin, but not actin (11). Likewise, some gastrointestinal stromal tumors may stain with calponin, but not with actin (10). Thus, we believe that calponin positivity in the spindled component of HFLL represents a population of myofibroblasts. Our HFLLs were negative for heavy caldesmon and desmin, ruling out smooth muscle phenotype. The location, morphology, and negativity for epithelial markers rule out myoepithelial differentiation. We further studied expression of calponin in CD34 positive tumors using control cases of spindle cell lipoma, dermatofibrosarcoma protuberans, and well-differentiated liposarcoma. These identified only a small subset of CD34 positive fibroblasts to coexpress calponin. Coexpression for calponin and CD34 was observed in all HFLL 


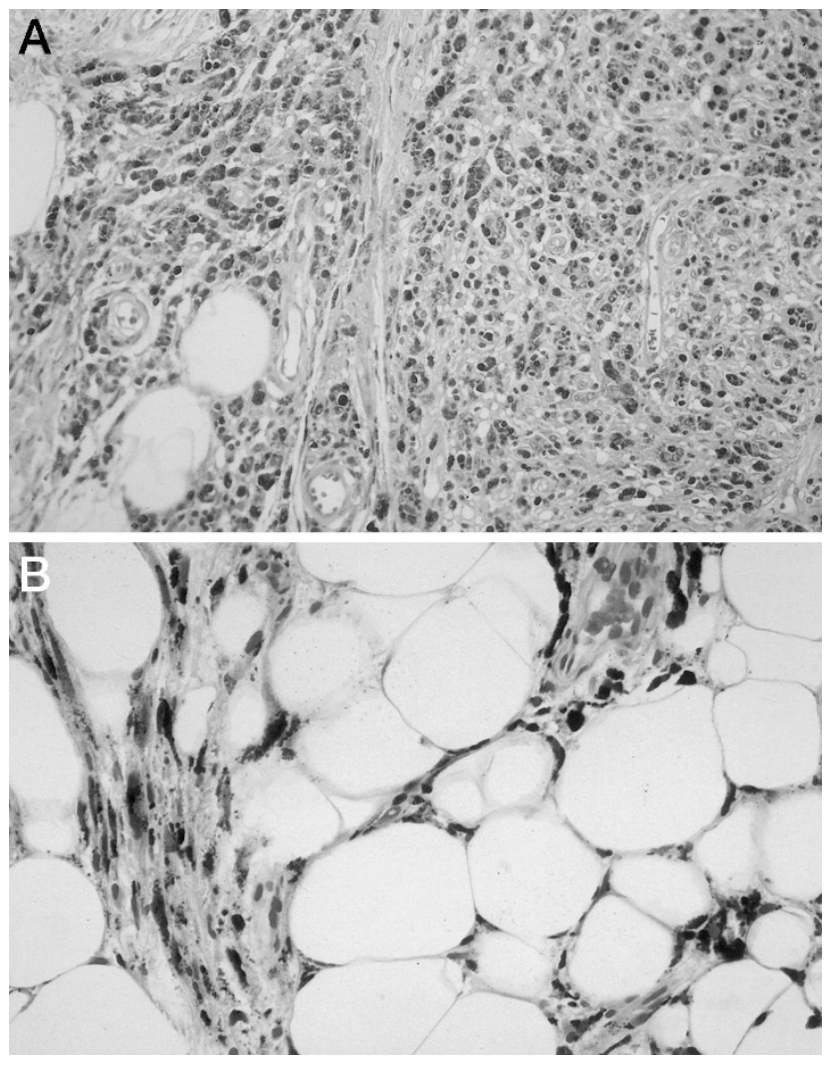

FIGURE 5. The pigment in HFLL has the granular golden appearance of iron (A); an iron stain (B), combined with a Fontana stain, confirms that this pigment is iron.

studied with these markers. This represents a unique immunohistochemical-staining pattern, and mixed cell population, requiring further investigation.

HFLL appears to be reactive. The superficial location, clinical history of antecedent trauma, lack of encapsulation (albeit circumscribed), homogeneously sized adipocytes, reactive appearance and mixed population of the spindled cells, hemosiderinladen macrophages, and inflammation suggest that this lesion is reactive rather than neoplastic. Similar to HFLL, local recurrence is reported in reactive lesions, such as nodular fasciitis (12).

Furthermore, we do not believe that HFLL is neoplastic because we have no evidence that HFLL is a form of a traumatized lipoma or other neoplastic process, despite its occasional extremely large size and local recurrence when incompletely excised. Superficial lipomas of the foot and ankle are rare but have been described in the literature (13), yet they are generally composed of heterogeneous fat cells. There is also no evidence that this represents an early phase of a developing spindle cell neoplasm because the recurrences of this entity did not exhibit more cellularity than the primary lesions. Fat was still a major component of the recurrence.

Some of our contributing pathologists considered various lipomatous lesions or fibrohistiocytic (myofibroblastic) in the differential diagnosis of HFLL.

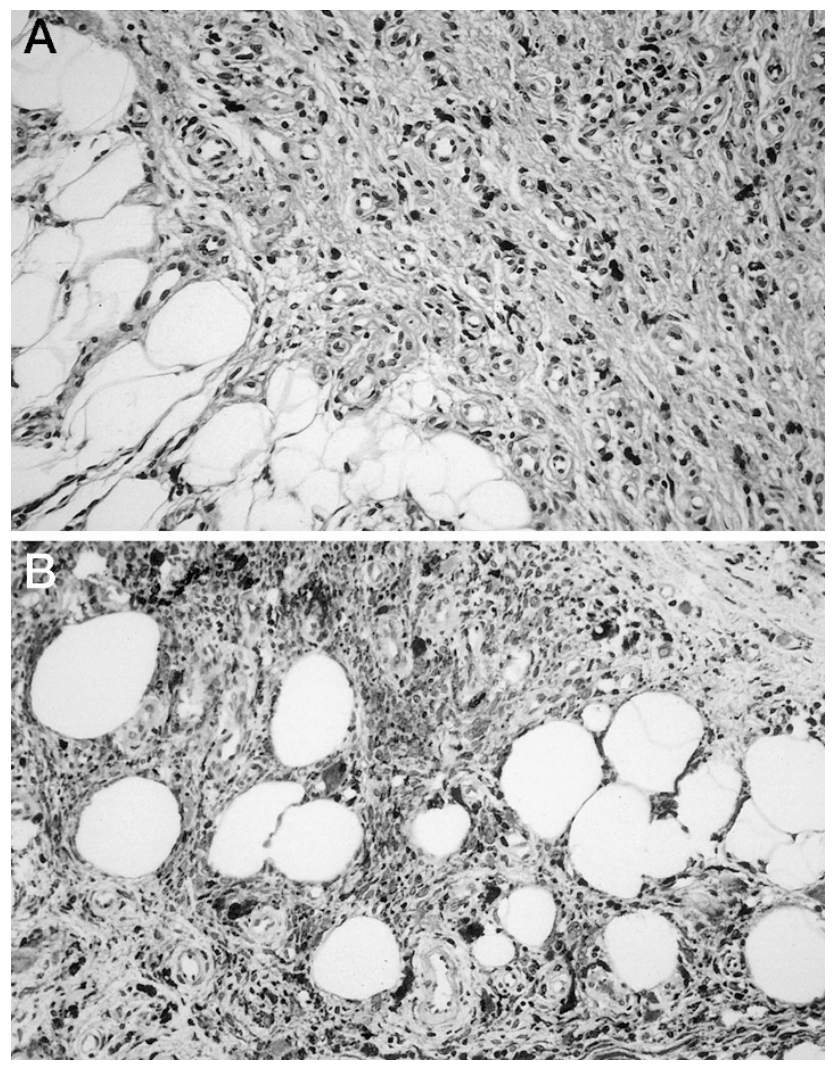

FIGURE 6. A KP-1 stain (A) and lysozyme (B) denote the histiocytic portion of the "fibrohistiocytic" mixture of the spindled areas in HFLL.

Although other spindle cell lesions may occur superficially in the foot or ankle in predominantly middle-aged women, the gross golden-brown color, abundant hemosiderin pigment, fibrohistiocytic morphology, mixed inflammation, and quilt-like pattern within abundant fat are unlike other known fibroblastic and lipomatous entities.

We believe that HFLL can be clinically and pathologically distinguished from (myo) fibroblastic and fibrohistiocytic lesions such as fibromatosis, nodular fasciitis, CD34 positive Kaposi-like diseases (acroangiodermatitis of Mali and Stewart-Bluefarb syndrome) (14), fibrous histiocytoma and its variants (15), pleomorphic hyalinizing angiectatic tumor of soft parts (16), and dermatofibrosarcoma protuberans (DFSP) because it is basically a lipomatous lesion with an abundant fatty component. Fibromatosis is characterized by uniform, bland myofibroblasts running in a purposeful direction with elongate vessels. It infiltrates but does not include fat within the lesion. It lacks the overall circumscription, pigment, plump and haphazard characteristics of the fibroblasts, and inflammation of HFLL. Nodular fasciitis, another reactive lesion, is similarly well-delineated, yet is composed of tissue-culture-like myofibroblasts arranged in short fascicular and storiform growth patterns admixed with foci of myxoid degeneration and extravasation of erythrocytes and lymphocytes. There is no fat 


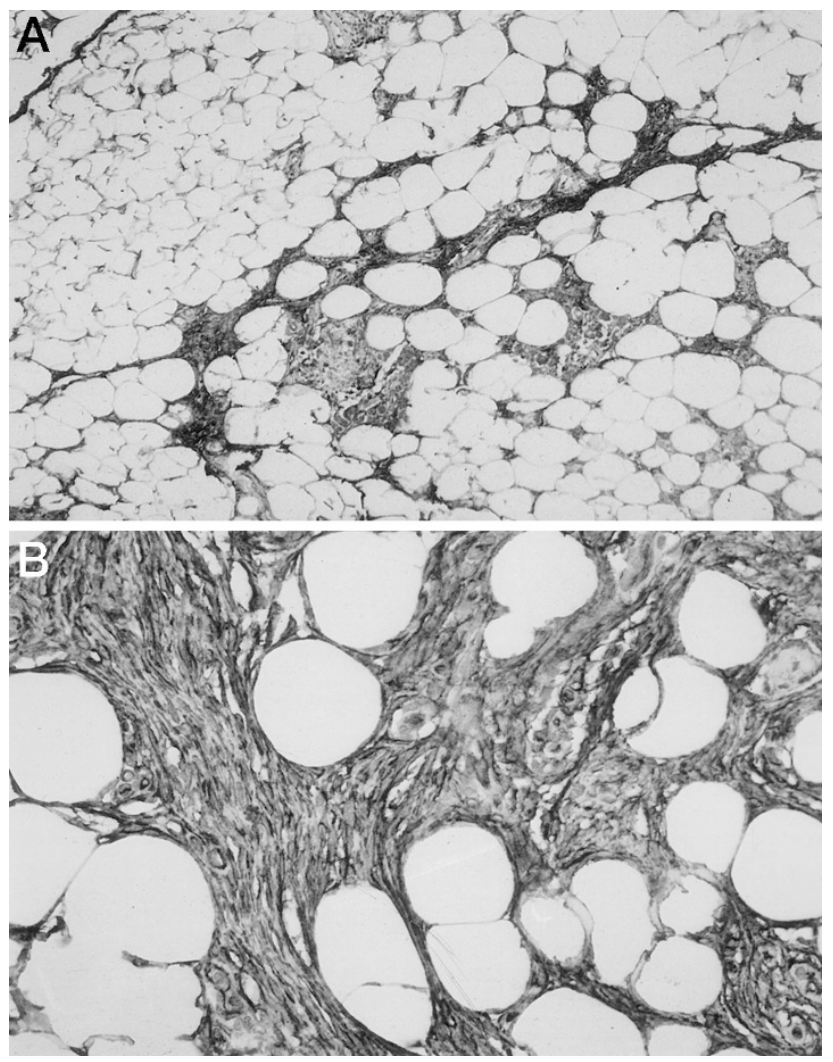

FIGURE 7. Calponin (A) and CD34 (B) reveal the mixture of myofibroblast and CD34-positive fibroblasts within the spindled component of HFLL.

within nodular fasciitis, and HFLL lacks the myxoid degeneration that is so characteristic of nodular fasciitis.

Sharing a common location of the distal lower extremity and similar morphologic characteristics (hemosiderin, spindled cells) with pseudo-Kaposi Mali (14) of the dermatopathology literature, HFLL is different in that it is subcutaneous rather than dermal and lacks superficial ulceration. The patients with HFLL are not known to have vascular insufficiency like those with pseudo-Kaposi lesions. Pseudo-Kaposi is thought to be caused by low blood flow to an area of trauma. It is hypothesized that vascular insufficiency causes retrograde blood flow with elevated venous and capillary pressures, oxygen saturation, and edema, stimulating the proliferation of capillaries and fibroblasts (granulationtype tissue). The elevated intravascular pressures probably result in extravasated erythrocytes and resultant hemosiderin deposition. It is possible that pseudo-Kaposi and HFLL, both with antecedent trauma and a presence of spindled cells and hemosiderin, could be caused by a common mechanism.

Another diagnostic consideration by contributing pathologists is fibrous histiocytoma, a tumor characterized by a dermal or subcutaneous proliferation of plump myofibroblasts in short fascicular and storiform patterns, with production of collagen, ad- mixed giant cells, foamy macrophages, and siderophages, a stellate periphery, and peripheral lymphocytes and plasma cells. The current lesion has similar features to the spindled cells of fibrous histiocytoma, yet the heavy iron pigment and quiltlike arrangement of the spindled cells within a circumscribed fatty component is different from the appearance of fibrous histiocytoma. The lipidized or "ankle-type" fibrous histiocytoma variant (15) has a similar ankle location to HFLL. However, the septal and perivascular proliferation of the myofibroblasts and fibroblasts, within the confines of a circumscribed lipomatous lesion in the latter lesion, is unlike the localized tumor growth of lipidized or "ankle-type" fibrous histiocytoma. Foam cells are not a component of HFLL, as they are in lipidized fibrous histiocytoma.

DFSP and pleomorphic hyalinizing angiectatic tumor (16) are characterized by similar CD34positive spindled cells as HFLL. Yet, DFSP has a monotonous, bland, spindle cell proliferation arranged in a storiform growth pattern that infiltrates but does not include fat in honeycomb and parallel growth patterns. Despite the apparent gross circumscription, DFSP is an infiltrative lesion. HFLL is a circumscribed fatty lesion. Unlike DFSP, the spindle cells of HFLL are more pleomorphic and are found in association with abundant iron pigment and scattered inflammatory cells.

Pleomorphic hyalinizing angiectatic tumor (PHAT) (16), also a lesion predominantly of the distal extremity subcutis in middle-aged females, has large ectatic spaces filled with blood, perivascular hyalinization, a spindled component that resembles a cross between malignant fibrous histiocytoma and neurofibroma, abundant intranuclear cytoplasmic inclusions, hemosiderin pigmentation, and occasional admixed fat. Despite the similar clinical setting, HFLL lacks the ectatic blood-filled spaces and pleomorphism of PHAT and is predominantly a fatty lesion. None of our cases exhibited intranuclear vacuolar inclusions. Although some of our cases represented the recurrent lesion, we did not identify any highly cellular areas reminiscent of pleomorphic hyalinizing angiectatic tumor of soft parts. Therefore, we do not consider HFLL as a precursor lesion to PHAT. PHAT is regarded as a low-grade malignant tumor, and our lesions all behaved in a benign manner.

Although some of our HFLLs were incorrectly diagnosed as possible known adipose tumors, hemosiderotic fibrohistiocytic lipomatous lesion can be distinguished from other fatty lesions, such as fat necrosis, fibrolipoma, spindle cell lipoma, spindle cell liposarcoma (17), myxoid liposarcoma, atypical lipomatous tumor, or well-differentiated liposarcoma. Fat necrosis is characterized by lipid macrophages that have finely vacuolated cytoplasm and 
centrally positioned nuclei that generally lacks heavy pigment deposition. Areas of fibrosis as well as inflammation and multinucleated giant cells are common. HFLL is a circumscribed lipocytic lesion with a quilt-like proliferation of myofibroblasts and fibroblasts rather than localized necrosis of normal fatty tissue. Fibrolipoma is a circumscribed benign lipoma with abundant collagen, typically found in the posterior neck region. HFLL lacks the presence of thick collagen bundles and exhibits more spindled cells and hemosiderin than fibrolipoma. Spindle cell lipoma, typically found on the neck or shoulder region of older males, contains uniform, bland spindle cells in a regular hatcheted or palisading pattern as well as mature fat, myxoid change, mast cells, and thick bundles of collagen fibers. Although they share many characteristics of spindle cell lipoma (mast cells, a mixture of fat, and spindled CD34 and calponin-positive cells), HFLLs may be distinguished from spindle cell lipomas by the pattern of spindle cell organization in HFLL, the plump pleomorphism of the spindle cells, the clinical location mainly on the distal extremity, the presence of heavy pigment, the lack of myxoid change, and the mixed inflammatory infiltrate. Spindle cell liposarcoma (17) is a variant of welldifferentiated liposarcoma that has spindled cells of different quality than that found in spindle cell lipoma, cytologic atypia, and/or lipoblasts. HFLL lacks the cytologic atypia found in that lesion and has characteristic hemosiderin pigment, a feature absent in spindle cell liposarcoma. Myxoid liposarcoma was proposed in the differential by one of the contributing pathologists, yet the myxoid features, delicate vasculature, and lipoblasts of that entity are absent in HFLL. Finally, HFLL does not demonstrate the cytologic atypia and widening of the fibrous septa that is seen in well-differentiated liposarcoma (atypical lipoma); HFLL has more pigment and mixed inflammation than are present in those lesions. We also could not demonstrate calponin positivity in the spindled component of seven welldifferentiated liposarcomas studied. However, careful examination of HFLL must be performed to exclude an underlying malignant lipomatous or fibroblastic lesion, such as well-differentiated liposarcoma or dermatofibrosarcoma protuberans.

\section{CONCLUSIONS}

In conclusion, HFLL is a rare, previously undescribed entity that typically occurs in the subcutis of the foot or ankle primarily of middle-aged women. It is composed of a reactive appearing perivascular, periadipocytic, and septal fibroblastic, histiocytic, and myofibroblastic proliferation within well-delineated fat. An inflammatory background, as well as abundant iron deposition, is always observed. The location, morphologic features, and prior history of local trauma suggest that these lesions are reactive. Despite slow growth, HFLL can reach a considerable size and have a potential for local recurrence, if incompletely excised.

\section{REFERENCES}

1. Enzinger FM, Weiss SW. Benign lipomatous tumors. In: Enzinger FM, Weiss SW, editors. Soft tissue tumors. 3rd ed. St. Louis, MO: Mosby; 1995. p. 381-430.

2. Weiss SW. Lipomatous tumors. Monogr Pathol. 1996;38:20739.

3. Silverman J, Tamsen A. Fibrohistiocytic differentiation in subcutaneous fatty tumors. Study of spindle cell, pleomorphic, myxoid, and atypical lipoma and dedifferentiated liposarcoma cases composed in part of CD34+ fibroblasts and FXIIIa + histiocytes. J Cutan Pathol 1997;23:484-93.

4. Suster S, Fisher C. Immunoreactivity for human hematopoietic progenitor cell antigen (CD34) in lipomatous tumors. Am J Surg Path 1997;21:195-200.

5. Weiss SW, Nickoloff BJ. CD-34 is expressed by a distinctive cell population in peripheral nerve, nerve sheath tumors, and related lesions. Am J Surg Pathol 1993;17:1039-45.

6. Hayashi K, Yano H, Hashida T, Takeuchi R, Takeda O, Asada $\mathrm{K}$, et al. Genomic structure of the human caldesmon gene. Proc Natl Acad Sci U S A 1992;89:12122-6.

7. Lazard D, Sastre X, Frid MG, Glukhova MA, Thiery JP, Koteliansky VE. Expression of smooth muscle-specific proteins in myoepithelium and stromal myofibroblasts of normal and malignant human breast tissue. Proc Natl Acad Sci U S A 1993;90:999-1003.

8. Miettinen M, Kovatich AJ, Sarlomo-Rikala M, Lasota J. Calponin and h-caldesmon in soft tissue tumors-consistent h-caldesmon immunoreactivity in gastrointestinal stromal tumors indicates traits of smooth muscle differentiation. Mod Pathol 1999;12:646-52.

9. Sobue K, Sellers JR. Caldesmon, a novel regulatory protein in smooth muscle and nonmuscle actomyosin systems. J Bio Chem 1991;266:12115-8.

10. Tazawa K, Tsukad K, Makuuchi H, Tsutsumi Y. An immunohistochemical and clinicopathological study of gastrointestinal stromal tumors. Pathol Int 1999;49:786-98.

11. Fanburg-Smith JC, Miettinen M. Angiomatoid. "malignant" fibrous histiocytoma: a clinicopathologic study of 158 cases and further exploration of the myoid phenotype. Hum Pathol 1999 Nov;30:1336-43.

12. Montgomery EA, Meis JM. Nodular fasciitis. Its morphologic spectrum and immunohistochemical profile. Am J Surg Pathol 1991;15:942-8.

13. Rydholm A, Berg N. Size, site and clinical incidence of lipoma. Factors in the differential diagnosis of lipoma and sarcoma. Acta Orthop Scand 1983;54:929-34.

14. Mali JW, Kuiper JP, Hamers AA. Acro-angiodermatitis of the foot. Arch Dermatol 1965;92:515-8.

15. Calonje E, Fletcher CDM. Cutaneous fibrohistiocytic tumors: an update. Adv Anat Pathol 1994;1:2-15.

16. Smith ME, Fisher C, Weiss SW. Pleomorphic hyalinizing angiectatic tumor of soft parts. A low-grade neoplasm resembling neurilemoma. Am J Surg Pathol 1996;20:21-9.

17. Dei Tos AP, Mentzel T, Newman PL, Fletcher CDM. Spindle cell liposarcoma, a hitherto unrecognized variant of liposarcoma. Analysis of six cases. Am J Surg Pathol 1994; 18:913-21. 